
\title{
$\begin{array}{ll}\text { Research Square } & \begin{array}{l}\text { Preprints are preliminary reports that have not undergone peer review. } \\ \text { They should not be considered conclusive, used to inform clinical practice, } \\ \text { or referenced by the media as validated information. }\end{array}\end{array}$
}

\section{RGL2 Drives the Metastatic Progression of Colorectal Cancer via Preventing the Protein Degradation of $\beta$-catenin and KRAS}

Meng-Shun Sun

Yuan's General Hospital

Lan-Ting Yuan

Yuan's General Hospital

Chia-Hao Kuei

Cardinal Tien Hospital

Hui-Yu Lin

Cardinal Tien Hospital

Yen-Lin Chen

Cardinal Tien Hospital

Hui-Wen Chiu

Taipei Medical University

Yuan-Feng Lin ( $\nabla$ d001089012@tmu.edu.tw )

Taipei Medical University College of Medicine

Research

Keywords: Colorectal cancer, metastasis, RGL2, Wnt/ $\beta$-catenin, KRAS

Posted Date: February 24th, 2021

DOI: https://doi.org/10.21203/rs.3.rs-229914/v1

License: (c) (i) This work is licensed under a Creative Commons Attribution 4.0 International License. Read Full License 


\section{Abstract}

Background: Colorectal cancer (CRC) is a common cancer and results in high mortality worldwide, owing to cancer progression, e.g., metastasis. However, the molecular mechanism underlying the metastatic evolution of CRC remains largely unknown. In this study, we investigated the clinical relevance and oncogenic function of RGL2 in CRC.

Methods: Cellular migration ability was assessed by transwell cultivation. The metastatic potential was estimated by lung colony-forming assay. DNA-binding activity of transcription factors was determined by luciferase-based reporter assay. RGL2 expression was analyzed by immunohistochemistry in human CRC specimens. RGL2 mRNA expression from the Cancer Genome Atlas (TCGA) CRC database was compared between the primary tumors and normal tissues.

Results: The upregulation of Ral Guanine Nucleotide Dissociation Stimulator Like 2 (RGL2) was commonly detected in primary tumors compared with normal tissues and is significantly associated with a poorer prognosis in CRC patients. Moreover, RGL2 expression appeared to positively correlate with the metastatic potential of CRC cells. Whereas RGL2 knockdown dramatically suppressed the metastatic potential of CRC cells in vitro and in vivo, RGL2 overexpression in poorly metastatic CRC cells and reconstitution in RGL2-silenced CRC cells enhanced and rescued the cellular metastatic ability, respectively. Computational simulation using the Gene Set Enrichment Analysis program and cell-based assays demonstrated that RGL2 expression was causally associated with the activity of the Wnt/ $\beta$ catenin signaling axis and KRAS, as well as the progression of epithelial-mesenchymal transition (EMT) in the detected CRC cells. Importantly, RGL2 upregulation was capable of preventing the protein degradation of $\beta$-catenin and KRAS in CRC cells.

Conclusion: RGL2 has metastasis-promoting effects via stabilizing $\beta$-catenin and KRAS in CRC cells and prognostic significance in CRC patients. These findings suggest that RGL2 acts as a driver to promote the metastatic progression of CRC and serves as a poor prognostic biomarker in CRC patients.

\section{Background}

Colorectal cancer (CRC) is the third most common cancer and the second leading cause of cancer-related death in Western countries, with more than 600,000 deaths worldwide each year [1]. Death resulting from $\mathrm{CRC}$ is associated with the disease stage, a more advanced grade, and the presence of obstruction [2]. Currently, two groups of CRC have been identified by molecular pathological characteristics [3;4]. The first group consists of approximately $16 \%$ of hypermutated tumors harboring microsatellite instability (MSI) due to defective mismatch repair (MMR). The second group belongs to nonhypermutated tumors ( 84\%) that are microsatellite stable (MSS) cancers with a high frequency of DNA somatic copy number alterations (SCNAs) and dysregulated Wnt pathway with frequent mutations in genes including adenomatous polyposis coli (APC) and Kirsten ras (KRAS). APC mutation is known to force the activation of $\beta$-catenin signaling cascades in CRC [5]. Several lines of evidence have shown that APC and KRAS 
mutations foster CRC progression, e.g., metastasis [6-9]. The interaction between $\beta$-catenin and mutant KRAS upon APC mutation was found to increase their protein stability and promote the metastatic progression of CRC [10]. Therefore, the simultaneous destabilization of $\beta$-catenin and mutant KRAS has been considered an effective anticancer strategy for CRC with APC mutations.

Ral guanine nucleotide dissociation stimulator-like (RGL) proteins consist of 4 subtypes, namely, RGL1, RGL2, RGL3 and RGL4. They are characterized as guanyl-nucleotide exchanging factors (GEFs) that regulate the exchange between GTP and GDP and are associated with the regulation of RAS activity [11]. A recent report showed that a decreased level of RGL4 is correlated with a poor prognosis and immune infiltration in lung adenocarcinoma [12]. In KRAS mutant pancreatic ductal adenocarcinoma (PDAC), an increased level of RGL2 was detected in tumors compared to normal tissues and was associated with PDAC metastatic capacity [13]. Nevertheless, the role of RGL2, as well as other RGLs, in KRAS-mediated CRC progression remains largely unknown.

Therefore, this study attempted to delineate the clinical relevance and oncogenic function of RGL2 in $\mathrm{CRC}$. We found that RGL2 upregulation is extensively detected in CRC compared to normal tissues and significantly correlated with a poorer prognosis in CRC patients. Moreover, RGL2 knockdown suppressed but overexpression promoted the metastatic potential of CRC cells in vitro and in vivo by fostering the progression of epithelial-mesenchymal transition and the activity of $\beta$-catenin. Importantly, our results suggest that RGL2 upregulation is capable of preventing the protein degradation of $\beta$-catenin and KRAS and thereby stabilizing the $\beta$-catenin and KRAS signaling pathways in metastatic CRC. This study is the first to document the oncogenic role of RGL2 in promoting cancer progression.

\section{Methods}

\section{Clinical and Molecular Data for CRC Patients}

The clinical data and overall survival (OS) time for the TCGA colorectal cancer patients were collected from the UCSC Xena website (UCSC Xena. Available online: http://xena.ucsc.edu/welcome-to-ucscxena/). The molecular data obtained by RNAseq (polyA p Illumina HiSeq) analysis of the TCGA colorectal cancer cohort were also downloaded from the UCSC Xena website. Microarray results with accession numbers GSE17536 and GSE17537 and the related clinical data were obtained from the Gene Expression Omnibus (GEO) database on the NCBI website. The raw intensities in the.CEL files were normalized by robust multichip analysis (RMA), and fold-change analysis was performed using GeneSpring GX11 (Agilent Technologies). Relative mRNA expression levels were normalized by the median of all samples and presented as $\log _{2}$ values.

\section{Cell lines and cell culture condition}

CRC cell lines DLD1, HT-29, HCT116 and LoVo were maintained in conditioned media supplemented with $10 \%$ fetal bovine serum (FBS) and incubated at $37^{\circ} \mathrm{C}$ with $5 \% \mathrm{CO}_{2}$. All cell lines were obtained from 
American Type Culture Collection (ATCC). All cells were routinely authenticated on the basis of short tandem repeat (STR) analysis, morphologic and growth characteristics and mycoplasma detection.

\section{Plasmid construction}

The gene encoding RGL2 was amplified from human cDNA (Invitrogen) using the standard polymerase chain reaction (PCR) procedure with paired primers and subcloned into the pDONR221 or pLAS3w vector. The pDONR221 plasmid was recombined with the destination vector pLenti6.3/V5-DEST in 293T cells to create packaged lentiviral particles. A commercially available plasmid pLenti/GFP sharing the same backbone (Invitrogen) was used as a control. The recombinant lentiviruses in the culture medium were harvested, concentrated using a Lenti-X Concentrator (Clontech), and then titrated by determining the viral RNA genome content with a Lenti-X qRT-PCR Titration kit (Clontech) according to the manufacturer's manual.

\section{Lentivirus-driven shRNA infection}

Lentiviral shRNA constructs were purchased from Open Biosystems. Lentiviruses were produced by cotransfecting the shRNA-expressing vector with the pMDG and $\mathrm{p} \triangle 8.91$ constructs into 293T cells using a calcium phosphate transfection kit (Invitrogen). After incubation for 48-72 hours, media were collected as viral stocks. Cells (50\% confluence) grown in 6 -well plates were bathed in fresh media containing 5 $\mu \mathrm{g} / \mathrm{ml}$ polybrene (Santa Cruz) before infection with a lentiviral viral particle-driven control or candidate gene shRNA at a multiplicity of infection (MOI) of 2-10 overnight. To select cells stably expressing the control or candidate gene shRNA, cells were further cultivated in the presence of puromycin $(10 \mu \mathrm{g} / \mathrm{ml})$ for 24 hours. Cell lystates from the puromycin-resistant cells were subsequently subjected to Western blot analysis to confirm the efficiency of gene knockdown.

\section{Reverse transcription PCR (RT-PCR)}

Total RNA was extracted from cells using a TRIzol extraction kit (Invitrogen). Aliquots ( $5 \mu \mathrm{g})$ of total RNA were treated with M-MLV reverse transcriptase (Invitrogen) and then amplified with Taq-polymerase (Protech) using paired primers (for RGL2, forward-GAAGAGGAGGAGGAAGAAGAAGAG and reverseGCTACCTCTGTTGTCCTCTCTAGTTC; for CDH1, forward-GGCACAGATGGTGTGATTACAGTC and reverseCAGGGTGAGAGAAGAGAGTGTATGT; for VIM, forward- CTGTGAAGTGGATGCCCTTAAAGG and reverseCAAGGTCATCGTGATGCTGAGAAG; for GAPDH, forward-AGGTCGGAGTCAACGGATTTG and reverseGTGATGGCATGGACTGTGGTC).

\section{Western blotting analysis}

Aliquots of total protein $(20-100 \mu \mathrm{g})$ from tested cell variants and HR Pre-Stained Protein Marker 10-170 $\mathrm{kDa}$ (BioTools, Taiwan) were separated by SDS-PAGE and then transferred to PVDF membranes. The membranes were subsequently incubated with blocking buffer ( $5 \%$ nonfat milk in TBS containing $0.1 \%$ Tween-20) for 2 hours at room temperature, primary antibodies against RGL2 (Genetex), phosphorylated (Ser33/37/Thr41) $\beta$-catenin/KRAS (Cell Signaling) and GAPDH (AbFrontier) overnight at $4^{\circ} \mathrm{C}$, and 
peroxidase-labeled species-specific secondary antibodies for 1 hour at room temperature. At each step, the membranes were extensively washed. Immunoreactive bands were finally visualized by an enhanced chemiluminescence system (Amersham Bioscience).

\section{Promoter reporter assay}

Cells grown on 12-well plates at $70 \%$ confluence at cell density were transiently transfected with the pGL4 [Luc2PCP/TCF/LEF-RE/Hygro] vector $(0.25 \mu \mathrm{g})$ using Lipofectamine 2000 (Invitrogen) according to the manufacturer's protocol. The p4.74-RLuc vector (Promega) at $0.0125 \mu \mathrm{g}$ was cotransfected as an internal control for transfection efficiency. After 24 hours of transfection, cells were lysed in Dual-GloTM Luciferase reagent. The firefly luciferase reaction was quenched by the addition of Dual-GLOTM Stop \& Glo $\circledast$ Reagent. The luciferase activities will be measured by chemiluminescence in a luminometer (Packard LumiCount ${ }^{\text {tM }}$ BL 10001) using the Dual-Glo ${ }^{\circledR}$ Luciferase Assay System (Promega) according to the manufacturer's protocol.

\section{Migration assay}

Polycarbonate filters were coated with human fibronectin on the lower side. Medium containing $10 \%$ FBS was added to each well of the lower compartment of the chamber. Cells were suspended in serum-free medium containing $0.1 \%$ FBS and loaded into each well of the upper chamber. After $16 \mathrm{~h}$, cells were fixed with methanol and then stained with Giemsa. Cells that migrated to the lower side of the membrane were counted under a light microscope $(\times 200$, ten random fields in each well). All experiments were performed in quadruplicate.

\section{Immunohistochemistry staining}

A paraffin-embedded tissue microarray of CRC purchased from SuperBioChips and 2 CRC sections obtained from Cardinal Tien Hospital in accordance with institutional review board approval (CTH-101-35-054) and the Declaration of Helsinki were heated and deparaffinized using xylene and rehydrated in a graded series of ethanol with a final wash in tap water. Antigen retrieval was performed using Retrieval Solution (DAKO) in a decloaking chamber (Biocare Medical). Endogenous peroxidase activity was quenched by hydrogen peroxide. Sections were then incubated with anti-RGL2 antibody (Genetex) at $4^{\circ} \mathrm{C}$ overnight. A Vectastain ABC peroxidase system (Vector Laboratories) was used to detect the reaction products.

\section{Animal experiment}

NOD/SCID mice were obtained from the National Laboratory Animal Center in Taiwan and were maintained in compliance with the institutional policy. All animal procedures were approved by the Institutional Animal Care and Use Committee at Taipei Medical University. For the in vivo lung metastatic colonization assay, $1 \times 10^{5}$ cells in $100 \mu$ of PBS were implanted into the mice through tail vein injection. Mice were humanely killed at the endpoint of experiments.

\section{Statistical analysis}


SPSS 17.0 software (Informer Technologies, Roseau, Dominica) was used to analyze the statistical significance. A paired t-test was utilized to compare Gah gene expression in the cancer tissues and corresponding normal tissues. Pearson's and nonparametric Spearman's correlation tests were performed to estimate the association among the mRNA levels of FOXD1, G3BP2, PL-D1 and TXNIP in the detected primary tumors. Evaluation of survival probabilities was determined by Kaplan-Meier analysis and logrank test. Student's t-test, paired t-test and one-way ANOVA with Tukey's test were used to estimate the statistical significance of the detected gene expression in clinical samples. The nonparametric Friedman test was used to analyze the nonparametric data. $p$ values $<0.05$ in all analyses were considered statistically significant.

\section{Results}

\section{RGL2 is upregulated in colorectal cancer compared to normal adjacent tissues}

We first analyzed the transcriptional profiling of RGL subtypes RGL1, RGL2, RGL3 and RGL4 in The Cancer Genome Atlas (TCGA) colorectal cancer (CRC) database. The data showed that the mRNA levels of RGL2 and RGL3 in primary tumors were significantly $(p<0.001)$ higher than those in normal tissues, whereas RGL1 and RGL2 were downregulated in primary tumors compared to normal tissues in the TCGA CRC database (Fig. 1a and b). The transcriptional profiling of RGL genes further demonstrated that the mRNA levels of RGL2, not RGL3, were significantly $(p<0.001)$ upregulated, but RGL1 and RGL4 were predominantly downregulated in primary tumors compared to normal adjacent colorectal tissues (Fig. 1c). Similar results were validated in the paired normal adjacent tissues and primary tumors derived from the GSE8671 CRC dataset (Fig. 1d). Based on these findings, we thereafter focused on investigating the clinical relevance and oncogenic role of RGL2 in CRC. We next performed immunohistochemistry (IHC) staining for RGL2 protein in sections derived from 2 CRC patients. The data revealed that the protein expression of RGL2 in the malignant areas was dramatically higher than that in the benign areas in the same section (Fig. 1e).

\section{RGL2 upregulation correlates with a poorer prognosis in CRC patients}

To evaluate the clinical relevance of RGL2, we next performed a meta-analysis of RGL2 transcripts against patients with different cancer types in the PrognoScan database. The data showed that RGL2 upregulation significantly $(p<0.05)$ correlated with an increased hazard ratio in the enrolled CRC cohorts, as well as 2 cohorts with blood and breast cancer (Fig. 2a). Kaplan-Meier analyses indicated that CRC patients with tumors harboring a higher RGL2 transcript exhibited poorer disease-free and overall survival rates in the GSE17537/GSE17536 CRC datasets (Fig. 2b) and TCGA CRC database (Fig. 2c), respectively. Moreover, another Kaplan-Meier analysis of RLG2 protein levels determined by IHC experiments (Fig. 2d) demonstrated that higher protein expression of RGL2 indicates a poorer overall survival probability in CRC patients enrolled in a commercial tissue microarray (Fig. 2e). Under the condition of overall survival 
probability, the data obtained from a Cox regression test using univariate and multivariate models demonstrated that RGL2 protein expression serves as an independent prognostic factor in CRC patients (Fig. 2f). A similar view was also found in the Cox repression test for RGL2 mRNA levels using overall survival conditions in the TCGA CRC patients (Table 1).

Table 1

Cox regression test under the condition of overall survival probability for RGL2 mRNA levels and clinical parameters, including age, gender, pathologic TNM (pTNM) stages, and tumor stage, against TCGA CRC cohort. The abbreviations HR, $\mathrm{Cl}$ and NA denote hazard ratio, confidence interval and not applicable, respectively.

\begin{tabular}{|c|c|c|c|c|}
\hline \multicolumn{5}{|c|}{ Overall survival $(n=184)$} \\
\hline Variables & Crude HR $(95 \% \mathrm{Cl})$ & $\mathrm{P}$ & Adjusted HR (95\%Cl) & $\mathrm{P}$ \\
\hline \multicolumn{5}{|l|}{ Age } \\
\hline$<64$ & 1 & NA & 1 & NA \\
\hline$\geq 64$ & $1.24(0.65-2.36)$ & 0.522 & $2.10(1.02-4.34)$ & 0.044 \\
\hline \multicolumn{5}{|l|}{ Gender } \\
\hline female & 1 & NA & 1 & NA \\
\hline male & $1.03(0.54-1.03)$ & 0.925 & $0.71(0.35-1.46)$ & 0.353 \\
\hline \multicolumn{5}{|l|}{ pT } \\
\hline T1-T2 & 1 & NA & 1 & NA \\
\hline T3-T4 & $4.27(1.03-17.8)$ & 0.046 & $1.90(0.42-8.67)$ & 0.409 \\
\hline \multicolumn{5}{|l|}{$\mathrm{pN}$} \\
\hline NO & 1 & NA & 1 & NA \\
\hline N1-N2 & $2.49(1.28-4.82)$ & 0.007 & $0.49(0.13-1.85)$ & 0.293 \\
\hline \multicolumn{5}{|l|}{ pM } \\
\hline MO & 1 & NA & 1 & NA \\
\hline M1 & $4.95(2.58-9.51)$ & $<0.001$ & $2.98(1.30-6.85)$ & 0.010 \\
\hline \multicolumn{5}{|l|}{ stage } \\
\hline$|-| \mid$ & 1 & NA & 1 & NA \\
\hline III-IV & $3.34(1.65-6.74)$ & 0.001 & $3.31(0.65-16.9)$ & 0.149 \\
\hline \multicolumn{5}{|c|}{ RGL2 expression } \\
\hline low & 1 & NA & 1 & NA \\
\hline high & $4.73(1.45-15.4)$ & 0.010 & $3.67(1.08-12.4)$ & 0.037 \\
\hline
\end{tabular}




\section{RGL2 upregulation promotes the metastatic progression of CRC cells}

We next examined the transcriptional profiling of RGL2 in primary tumors derived from the TCGA CRC patients with different pathologic $\mathrm{N}$ stages. The data showed that RGL2 upregulation highly correlated with the progression of lymph node metastasis in CRC patients (Fig. 3a). Similarly, we found that the endogenous mRNA and protein levels of RGL2 (Fig. 3b) were causally associated with cellular migration ability (Fig. 3c) in the detected CRC cell lines. The gene knockdown of RGL2 with 3 independent shRNA clones dramatically reduced RGL2 mRNA and protein levels (Fig. 3d) and cellular migration ability (Fig. 3e and f) compared to the control groups in HT-29 cells that express a relatively higher mRNA and protein levels of RGL2 (Fig. 3b) and stronger migration ability (Fig. 3c) in a panel of CRC cell lines. Importantly, RGL2 knockdown also significantly $(p<0.001)$ suppressed the lung colony-forming ability of HT-29 cells in the animal experiments (Fig. $3 g$ and $h$ ). Conversely, the enforced expression of the exogenous RGL2 gene predominantly elevated the intracellular mRNA and protein levels of RGL2 (Fig. 4a) and enhanced the cellular migration ability (Fig. 4b and c) compared with the control cell variants in HCT116 cells that harbored relatively lower RGL2 expression and exhibited a poorer migration ability in the detected CRC cells. The reconstitution of RGL2 expression by transiently transfecting an exogenous RGL2 gene into RGL2-silenced HT-29 cells showed that the repressed mRNA and protein levels of RGL2 (Fig. 4d) and the suppressed cellular migration ability (Fig. $4 \mathrm{e}$ and f) were robustly restored. In addition, RGL2 overexpression predominantly fostered the lung metastatic ability of HCT116 cells (Fig. $4 \mathrm{~g}$ and h). These findings suggest a driver function of RGL2 in promoting the metastatic progression of CRC.

\section{RGL2 induces the activation of the Wnt/ $\beta$-catenin pathway and EMT progression and thereby promotes CRC metastasis}

To ascertain the possible mechanism for RGL2-promoted CRC metastasis, we performed Spearman's correlation test against RGL2 coexpression with other somatic genes detected by RNA-sequencing experiments in the TCGA CRC samples with higher RGL2 expression in Kaplan-Meier analysis and a record of lymph node metastasis (Fig. 5a). Then, we used the ranked Spearman's coefficient $\rho$ values (Fig. 5b) as the RGL2 signature to perform a computer simulation using the Gene Set Enrichment Analysis (GSEA) program against the Hallmark gene set deposited in the Molecular Signature Database. GSEA results revealed that the obtained RGL2 signature highly correlated with the activation of the Wnt/ $\beta$-catenin pathway and EMT progression in the enrolled TCGA CRC samples (Fig. $5 c$ and d). A luciferasebased promoter assay demonstrated that RGL2 knockdown significantly $(p<0.001)$ diminished the binding of $\beta$-catenin to the TCF-LEF response element within the upstream promoter region of the luciferase gene and thereby suppressed luciferase activity in HT-29 cells compared to control cell variants (Fig. 5e). In contrast, RGL2 overexpression robustly elevated the luciferase activity owing to the enhanced DNA binding of $\beta$-catenin in the examined HCT116 cells compared to the control cell variants (Fig. $5 f$ ). 
The reconstitution of RGL2 expression in the RGL2-silenced HT-29 cells predominantly rescued the DNA binding of $\beta$-catenin, as shown by strongly elevated luciferase activity in the detected HT-29 cell variant compared to the control cells (Fig. 5g). Moreover, RT-PCR results demonstrated that RGL2 knockdown dramatically suppressed EMT progression, as judged by enhanced CDH1 (E-cadherin) levels but repressed VIM (vimentin) expression in HT-29 cells (Fig. 5h), whereas RGL2 overexpression potentiated EMT progression, as shown by reduced $\mathrm{CDH} 1$ expression but an increased level of VIM (Fig. 5i). Accordingly, the reconstitution of RGL2 expression in RGL2-silenced HT-29 cells reinforced EMT progression, as determined by repressed CDH1 expression and an enhanced level of VIM (Fig. 5j).

\section{RGL2 upregulation enhances the protein stability of $\beta$ - catenin and KRAS in metastatic CRC cells}

Approximately $50 \%$ of CRC carries a KRAS mutation, while approximately $90 \%$ and $5 \%$ of CRC harbor mutations in $A P C$ and $\beta$-catenin, respectively. KRAS, $A P C$ and $\beta$-catenin mutations in colon cancers have been associated with poorer survival and increased tumor aggressiveness [10]. We next dissected the transcriptional profiling of RGL2 in the TCGA CRC samples without or with APC, $\beta$-catenin and KRAS mutations. The data showed that RGL2 mRNA levels were not significantly different between CRC samples harboring wild-type and mutant $A P C$, $\beta$-catenin and KRAS genes (Fig. 6a-C). Intriguingly, RGL2 knockdown dramatically suppressed the protein levels of KRAS in highly metastatic HT-29 cells (Fig. 6d), whereas RLG2 overexpression predominantly enhanced the protein expression of KRAS in poorly metastatic HCT116 cells (Fig. 6e). Moreover, the reconstitution of RGL2 expression in RGL2-silenced HT29 cells robustly restored the intracellular protein levels of KRAS (Fig. 6f). We next pretreated HT-29 cells without or with RGL2 knockdown and HCT116 cells without or with RGL2 overexpression with cycloheximide, an inhibitor of protein synthesis, at $10 \mu \mathrm{M}$ for the designated time periods prior to performing Western blot analyses to determine whether the RGL2-enhanced protein expression of KRAS is associated with the posttranslational modification machinery. The data showed that RGL2 knockdown accelerated the protein degradation of KRAS in the cyloheximide-treated HT-29 cell variants (Fig. 6g); conversely, RGL2 overexpression extended the protein expression of KRAS in the cyloheximide-treated HCT116 cell variants (Fig. 6h). Since a previous report has shown that the interaction of KRAS with $\beta$ catenin prevents the protein degradation of $\beta$-catenin [14], we further examined the protein levels of $\beta$ catenin in the presence of cyloheximide in the HT-29 and HCT116 cell variants. The data showed that RGL2 knockdown in HT-29 cells (Fig. 6g) promoted but RGL2 overexpression in HCT116 cells (Fig. 6h) compromised the protein degradation of $\beta$-catenin, as determined by the protein levels of phosphorylated $\beta$-catenin at Ser33/37/Thr41. The phosphorylation of Ser33/37/Thr41 residues has been shown to be critical for $\beta$-catenin degradation [15]. Based on our findings, we proposed that RGL2 upregulation promotes CRC metastasis by preventing the protein degradation of KRAS and $\beta$-catenin (Fig. 6i).

\section{Discussion}

The prevalence of KRAS mutations in comparison with the prevalence of the NRAS and HRAS isoforms is relatively higher in CRC [16]. KRAS mutations have been correlated with malignant progression, e.g., drug 
resistance and metastasis in CRC. Here, we found that RGL2 expression is not relevant to the KRAS mutation in the TCGA CRC patients. Furthermore, RGL2 is upregulated in CRC compared to normal adjacent tissues. RGL2 upregulation correlated with a poorer prognosis in CRC patients. The current study is the first to demonstrate the clinical relevance of RGL2 in CRC. A previous study observed the upregulation of RGL2 expression in the tumor tissue and cell lines of pancreatic ductal adenocarcinoma (PDAC). Interfering RNA suppression of RGL2 reduced steady-state Ral activity, growth and invasion in PDAC cells [13]. The downstream effectors of Ras proteins, including Ras-related protein Ral-A (RalA) and Ras-related protein Ral-B (RaIB), are activated by RaIGEF. The RaIGEF-Ral signaling pathway caused Rasinduced transformation of human cells [17]. RGL2, which is one of six RalGEFs, harbors Ras-binding domains and directly signals downstream Ras proto-oncogenes toward Ral GTPases [18]. Santos et al. indicated that silencing RGL2 inhibited anchorage-dependent and independent cell growth in human nonsmall cell lung carcinoma (NSCLC). Moreover, the inhibition of RalGEF with PH domain and SH3 domainbinding motif 2 (RaIGPS2), which is a Ras-independent RaIGEF, induced apoptosis and cell cycle arrest in NSCLC cells. In addition, RaIGPS2 affected several important cell cycle regulators, including the E3 ubiquitin protein ligase S-phase kinase-associated protein 2 (Skp2) and the cell cycle inhibitors p27 and p21 [19].

Recent evidence shows that inhibition of RalA expression suppresses the transformation and growth of human pancreatic cancer cells. Furthermore, RalB was required for metastasis and invasion in vitro and in vivo [20]. Active Ras signals to RalB by RGL1 and RGL2 promote invasiveness, and the contribution of RalB may be more crucial than that of the PI3K and MAPK pathways. In addition, RalB expression at the protein level in breast cancer increased in a manner consistent with progression toward metastasis [21]. Dysregulation of motility and adhesion of cells are important events in the development of metastasis. Another recent study concluded that CycD1-Cdk4 enhanced cell motility and detachment by increasing the phosphorylation of RGL2 [22]. In the present study, RGL2 upregulation promoted the metastatic progression of CRC cells. RGL2 knockdown significantly suppressed the lung colony-forming ability of HT-29 cells in animal experiments. Conversely, forced expression of the exogenous RGL2 gene enhanced the cellular migration ability of CRC cells. GSEA results revealed that the obtained RGL2 signature highly correlates with EMT progression in the TCGA CRC samples. RGL2 knockdown dramatically suppresses EMT progression by enhancing $\mathrm{CDH} 1$ levels but represses VIM expression in HT-29 cells. However, the mechanisms through which RGL2 enhances metastasis in CRC are unclear. Hyperactivation of Wnt/ $\beta$ catenin signaling is a common feature in most CRC patients, and this pathway is an important regulator of CRC development and metastasis [23]. We found that RGL2 promoted CRC metastasis via activation of the Wnt/ $\beta$-catenin pathway.

In our current study, RGL2 expression was irrelevant to KRAS mutation but regulated the protein levels of KRAS in CRC. Furthermore, the RGL2-enhanced protein expression of KRAS is associated with the posttranslational modification machinery. A previous report demonstrated that the interaction of KRAS with $\beta$-catenin inhibited the protein degradation of $\beta$-catenin [14]. RGL2 knockdown in HT-29 cells promoted the protein degradation of $\beta$-catenin. The direct interaction of $\beta$-catenin with RAS protein has been shown to promote RAS degradation by glycogen synthase kinase 3 beta (GSK3 $\beta$ )-mediated 
polyubiquitination-dependent proteasomal degradation in HCT116 cells harboring wild-type $\beta$-catenin [14]. GSK3 $\beta$ is a key component of the $\beta$-catenin destruction complex. Costabilization of RAS and $\beta$ catenin, particularly the KRAS mutant form, increased the growth of CRC, and high levels of RAS and $\beta$ catenin were found in CRC patient tissues [24;25]. The degradation of $\beta$-catenin and RAS provides pathological significance and a mechanical basis for the enhancement of colorectal tumorigenesis [14]. Here, we found that RGL2 expression affected the protein stability of KRAS in CRC cells. RGL2 upregulation enhances the protein stability of $\beta$-catenin and KRAS in metastatic CRC cells.

\section{Conclusion}

We identified that RGL2 is upregulated in primary tumors compared to normal tissues derived from CRC patients. RGL2 upregulation is related to poorer prognosis in CRC patients. Moreover, RGL2 expression was associated with the metastatic potential of CRC cells. RGL2 promoted the metastatic progression of CRC cells. In addition, RGL2 induced the activation of the Wnt/ $\beta$-catenin pathway and EMT progression and thereby promoted CRC metastasis. This is the first study to document that RGL2 is capable of stabilizing KRAS and $\beta$-catenin signaling in CRC. These findings suggest that RGL2 promotes the metastasis of CRC and serves as a poor prognostic biomarker in CRC.

\section{Abbreviations}

CRC: colorectal cancer; RGL2: Ral Guanine Nucleotide Dissociation Stimulator Like 2; TCGA: The Cancer Genome Atlas; EMT: epithelial-mesenchymal transition; DFS: disease-free survival; OS: overall survival; OE: overexpression; KD: knockdown; NS: non-silencing; VC: vector control; GSK3 $\beta$ : glycogen synthase kinase 3 beta; VIM: vimentin; GSEA: Gene Set Enrichment Analysis; APC: adenomatous polyposis coli; KRAS: Kirsten ras.

\section{Declarations}

\section{Acknowledgements}

Not applicable.

\section{Authors' Contributions}

Conception and design: M.-S.S., L.-T.Y., C.-H.K., H.-Y.L., H.-W.C. and Y.-F.L. Acquisition of data: M.-S.S., L.T.Y., Y.-L.C. and H.-W.C. Analysis and interpretation of data: M.-S.S., H.-W.C. and Y.-F.L. Writing, review and/or revision of the manuscript: M.-S.S., H.-W.C. and Y.-F.L. All authors read and approved the final manuscript.

\section{Funding}


This study was supported by the Ministry of Science and Technology, Taiwan (MOST 108-2320-B-038017-MY3 to Yuan-Feng Lin; MOST 109-2314-B-038-078-MY3 to Hui-Wen Chiu) and Yuan's General Hospital/Taipei Medical University (107YGH-TMU-02).

\section{Availability of data and materials}

Data available in a publicly accessible repository that does not issue DOls. Publicly available datasets were analyzed in this study. This data can be found here: [https://www.ncbi.nlm.nih.gov/geo/query/ acc.cgi?acc=GSE17536/accession number: GSE17536], [https://www.ncbi.nlm.nih.gov/geo/query/ acc.cgi?acc=GSE17537/accession number: GSE17537].

\section{Ethics approval and consent to participate}

The study was reviewed and approved by the Institutional Review Board (IRB) and the Office of Human Research Ethics of the Cardinal Tien Hospital at Taiwan. All patients signed an informed consent before the surgery. All animals' experimental procedures were approved by Institutional Animal Care and Use Committee of Taipei Medical University at Taiwan.

\section{Consent for publication}

Not applicable.

\section{Competing interests}

The authors declare no conflict of interest.

\section{References}

1. Ferlay J, Steliarova-Foucher E, Lortet-Tieulent J, Rosso S, Coebergh JW, Comber H, et al. Cancer incidence and mortality patterns in Europe: estimates for 40 countries in 2012. Eur.J.Cancer 2013; 49:1374-1403.

2. Griffin MR, Bergstralh EJ, Coffey RJ, Beart RW, Jr., Melton LJ, III. Predictors of survival after curative resection of carcinoma of the colon and rectum. Cancer 1987; 60:2318-2324.

3. Comprehensive molecular characterization of human colon and rectal cancer. Nature 2012; 487:330337.

4. Guinney J, Dienstmann R, Wang X, de RA, Schlicker A, Soneson C, et al. The consensus molecular subtypes of colorectal cancer. Nat.Med. 2015; 21:1350-1356. 
5. Morin PJ, Sparks AB, Korinek V, Barker N, Clevers H, Vogelstein B, et al. Activation of beta-catenin-Tcf signaling in colon cancer by mutations in beta-catenin or APC. Science 1997; 275:1787-1790.

6. Park HS, Chun YJ, Kim HS, Kim JH, Lee CK, Beom SH, et al. Clinical features and KRAS mutation in colorectal cancer with bone metastasis. Sci.Rep. 2020; 10:21180.

7. Chu PC, Lin PC, Wu HY, Lin KT, Wu C, Bekaii-Saab T, et al. Mutant KRAS promotes liver metastasis of colorectal cancer, in part, by upregulating the MEK-Sp1-DNMT1-miR-137-YB-1-IGF-IR signaling pathway. Oncogene 2018; 37:3440-3455.

8. Sakai E, Nakayama M, Oshima H, Kouyama Y, Niida A, Fujii S, et al. Combined Mutation of Apc, Kras, and Tgfbr2 Effectively Drives Metastasis of Intestinal Cancer. Cancer Res. 2018; 78:1334-1346.

9. Chen J, Rocken C, Lofton-Day C, Schulz HU, Muller O, Kutzner N, et al. Molecular analysis of APC promoter methylation and protein expression in colorectal cancer metastasis. Carcinogenesis 2005; 26:37-43.

10. Jeong WJ, Ro EJ, Choi KY. Interaction between Wnt/beta-catenin and RAS-ERK pathways and an anti-cancer strategy via degradations of beta-catenin and RAS by targeting the Wnt/beta-catenin pathway. NPJ.Precis.Oncol. 2018; 2:5.

11. Hofer F, Fields S, Schneider C, Martin GS. Activated Ras interacts with the Ral guanine nucleotide dissociation stimulator. Proc.Natl.Acad.Sci.U.S.A 1994; 91:11089-11093.

12. Sun $Y$, Zhang $Y$, Ren S, Li X, Yang P, Zhu J, et al. Low expression of RGL4 is associated with a poor prognosis and immune infiltration in lung adenocarcinoma patients. Int.Immunopharmacol. 2020; 83:106454.

13. Vigil D, Martin TD, Williams F, Yeh JJ, Campbell SL, Der CJ. Aberrant overexpression of the Rgl2 Ral small GTPase-specific guanine nucleotide exchange factor promotes pancreatic cancer growth through Ral-dependent and Ral-independent mechanisms. J.Biol.Chem. 2010; 285:34729-34740.

14. Lee SK, Jeong WJ, Cho YH, Cha PH, Yoon JS, Ro EJ, et al. beta-Catenin-RAS interaction serves as a molecular switch for RAS degradation via GSK3beta. EMBO Rep. 2018; 19.

15. Wu G, He X. Threonine 41 in beta-catenin serves as a key phosphorylation relay residue in betacatenin degradation. Biochemistry 2006; 45:5319-5323.

16. Cox AD, Fesik SW, Kimmelman AC, Luo J, Der CJ. Drugging the undruggable RAS: Mission possible? Nat.Rev.Drug Discov. 2014; 13:828-851.

17. Hamad NM, Elconin JH, Karnoub AE, Bai W, Rich JN, Abraham RT, et al. Distinct requirements for Ras oncogenesis in human versus mouse cells. Genes Dev. 2002; 16:2045-2057.

18. Ceriani M, Scandiuzzi C, Amigoni L, Tisi R, Berruti G, Martegani E. Functional analysis of RalGPS2, a murine guanine nucleotide exchange factor for RalA GTPase. Exp.Cell Res. 2007; 313:2293-2307.

19. Santos O, Parrini MC, Camonis J. RalGPS2 Is Essential for Survival and Cell Cycle Progression of Lung Cancer Cells Independently of Its Established Substrates Ral GTPases. PLoS.One. 2016; 11:e0154840. 
20. Lim KH, O'Hayer K, Adam SJ, Kendall SD, Campbell PM, Der CJ, et al. Divergent roles for RalA and RalB in malignant growth of human pancreatic carcinoma cells. Curr.Biol. 2006; 16:2385-2394.

21. Zago G, Veith I, Singh MK, Fuhrmann L, De BS, Remorino A, et al. RalB directly triggers invasion downstream Ras by mobilizing the Wave complex. Elife. 2018; 7.

22. Fernandez RM, Ruiz-Miro M, Dolcet X, Aldea M, Gari E. Cyclin D1 interacts and collaborates with Ral GTPases enhancing cell detachment and motility. Oncogene 2011; 30:1936-1946.

23. Cheriyamundath S, Ben-Ze'ev A. Wnt/beta-Catenin Target Genes in Colon Cancer Metastasis: The Special Case of L1CAM. Cancers (Basel). 2020; 12.

24. Jeong WJ, Yoon J, Park JC, Lee SH, Lee SH, Kaduwal S, et al. Ras stabilization through aberrant activation of Wnt/beta-catenin signaling promotes intestinal tumorigenesis. Sci.Signal. 2012; 5:ra30.

25. Moon BS, Jeong WJ, Park J, Kim TI, Min dS, Choi KY. Role of oncogenic K-Ras in cancer stem cell activation by aberrant Wnt/beta-catenin signaling. J.Natl.Cancer Inst. 2014; 106:djt373.

\section{Figures}


A TCGA_CRC gene expression $(\mathrm{N}=431)$ B
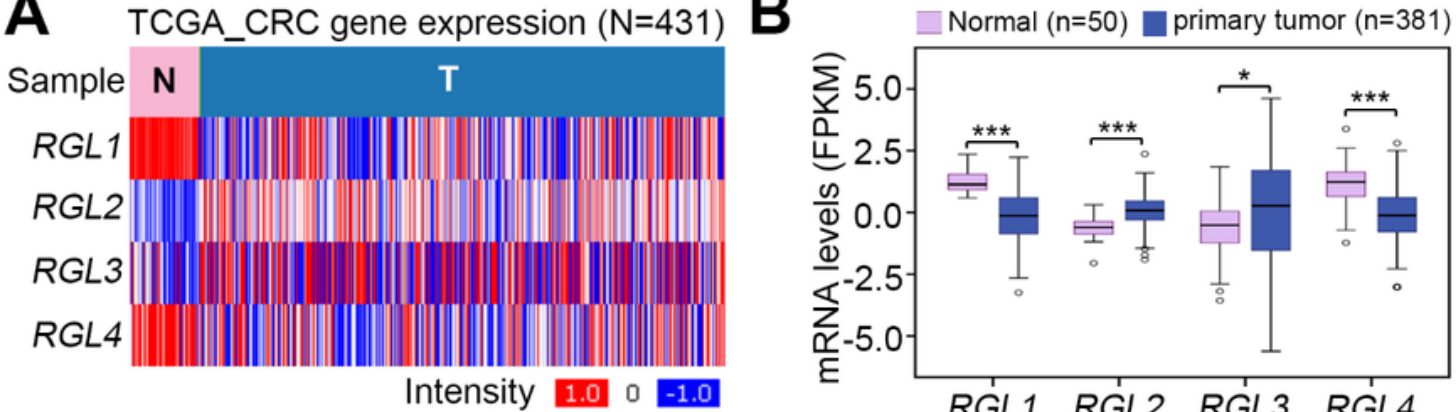

C

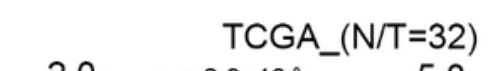

RGL1 RGL2 RGL3 RGL4
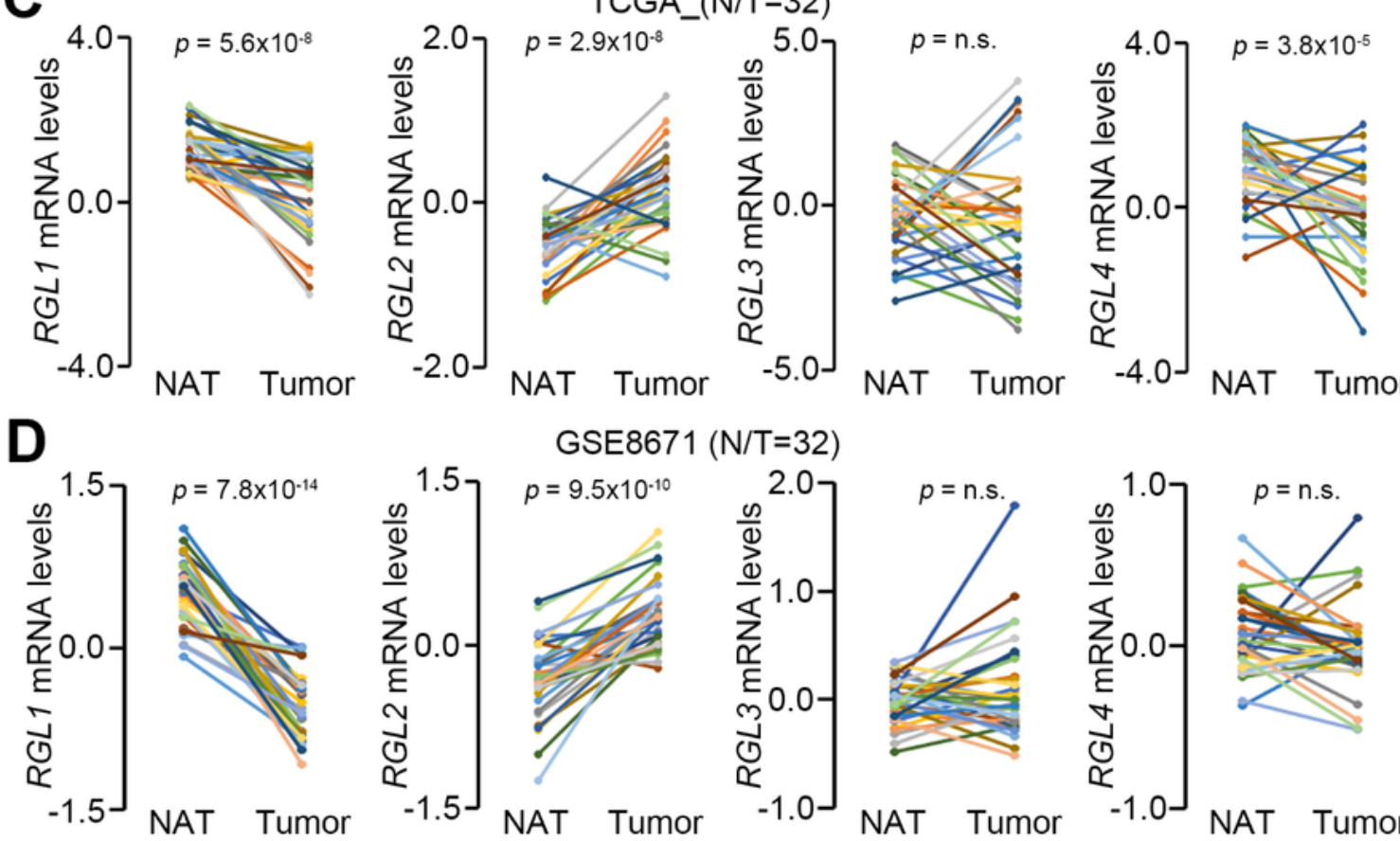

$(\mathrm{N} / \mathrm{T}=32)$

E

Patient \#1
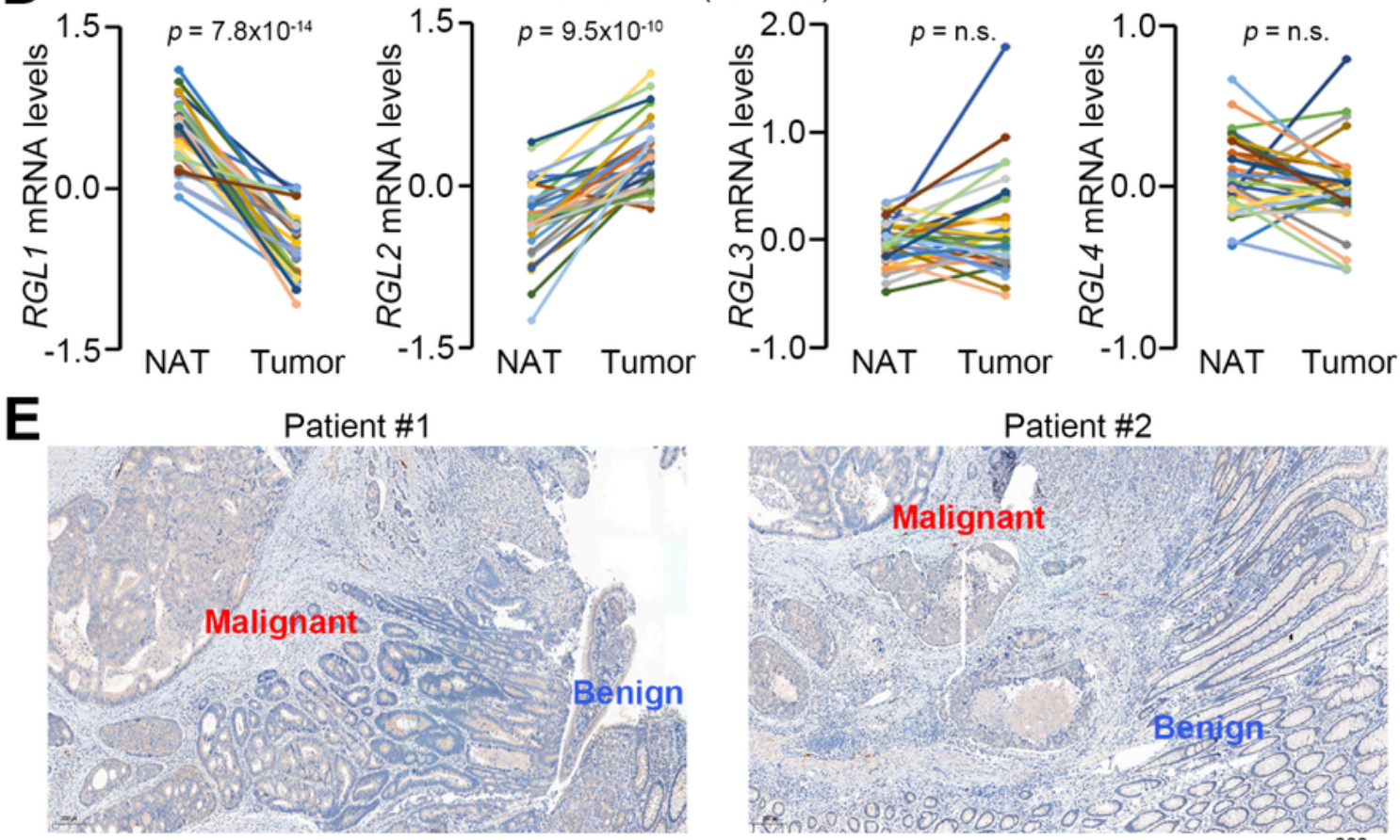

Patient \#2

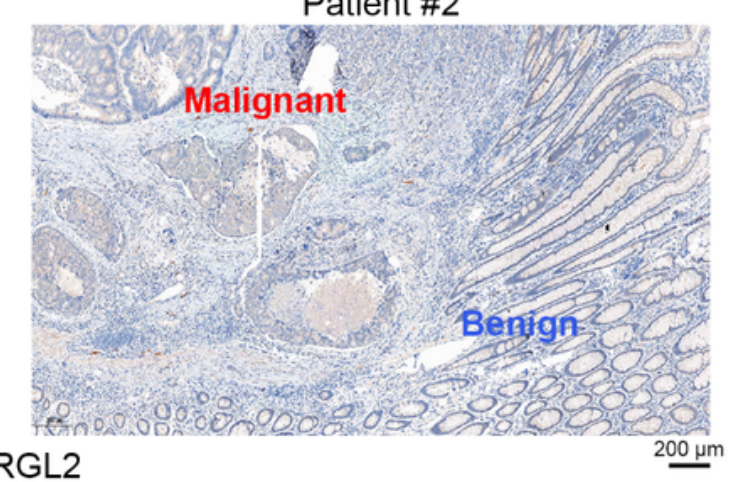

\section{Figure 1}

RGL2 is upregulated in primary tumors compared to normal tissues derived from colorectal cancer patients. ( $a$ and b) Heatmap ( $a$ ) and boxplots (b) for the transcription profiling of RGL1, RGL2, RLG3 and RGL4 in the TCGA colorectal cancer database. The statistical significance was analyzed by t-test. (c and d) The mRNA levels of RGL1, RGL2, RLG3 and RGL4 in normal adjacent tissues (NATs) and primary tumors from the TCGA (c) and GSE8671 oral colorectal cancer patients (d). The statistical significance 
was evaluated by paired t-tests. (e) IHC staining for RGL2 protein in 2 representative colorectal cancer patients. The malignant and benign areas were defined by the morphology of tumors.

A

$\begin{array}{llllll}\text { DATASET } & \text { CANCER } & \text { ENDPOINT } & \text { ARRAY } & \text { PROBE } & \text { N } \\ \text { GSE12417 } & \text { Blood } & \text { OS } & \text { HG-U133_2 } & 209110 & 79 \\ \text { GSE12417 } & \text { Blood } & \text { OS } & \text { HG-U133A } & 209110 & 163 \\ \text { GSE3143 } & \text { Breast } & \text { OS } & \text { HG-U95A } & 40521 & 158 \\ \text { GSE12276 } & \text { Breast } & \text { RFS } & \text { HG-U133_2 } & 209110 & 204 \\ \text { E-TABM-158 } & \text { Breast } & \text { DMFS } & \text { HG-U133A } & 209110 & 117 \\ \text { E-TABM-158 } & \text { Breast } & \text { DSS } & \text { HG-U133A } & 209110 & 117 \\ \text { GSE17537 } & \text { Colorectal } & \text { DFS } & \text { HG-U133_2 } & 209110 & 55 \\ \text { GSE17536 } & \text { Colorectal } & \text { DFS } & \text { HG-U133-_2 } & 209110 & 145 \\ \text { jacob-00182 } & \text { Lung } & \text { OS } & \text { HG-U133A } & 209110 & 104 \\ \text { GSE31210 } & \text { Lung } & \text { RFS } & \text { HG-U133_2 } & 209110 & 204\end{array}$

\section{PrognoScan Database}

OS: overall survival; RFS: relapse-free survival; DMFS: distant metastasis-free survival;

B
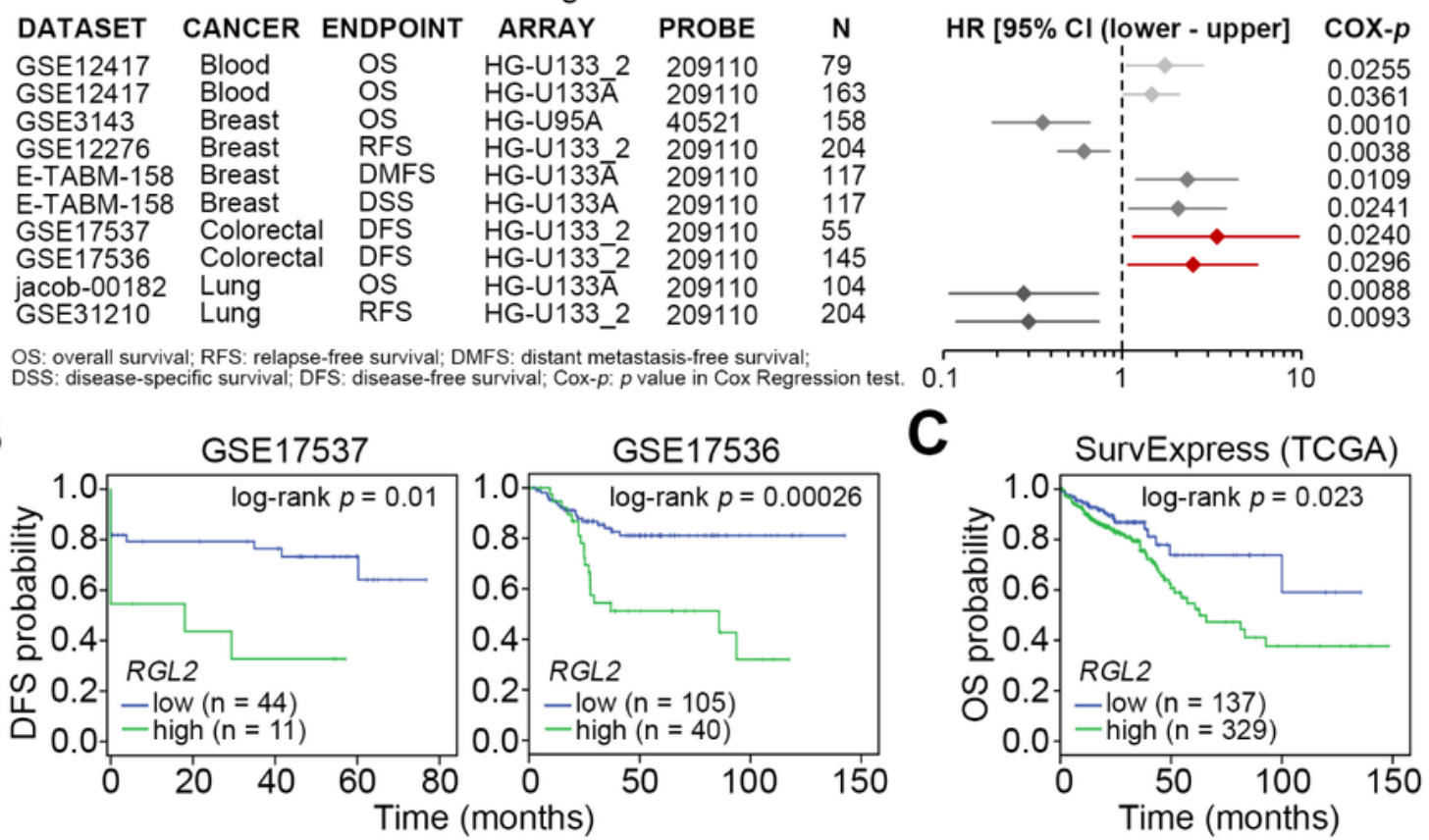

D

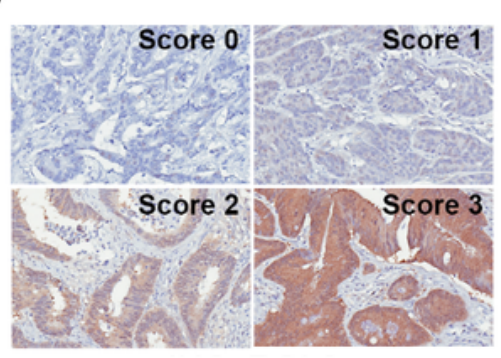

IHC: RGL2

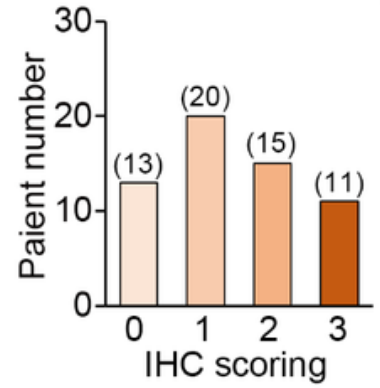

E

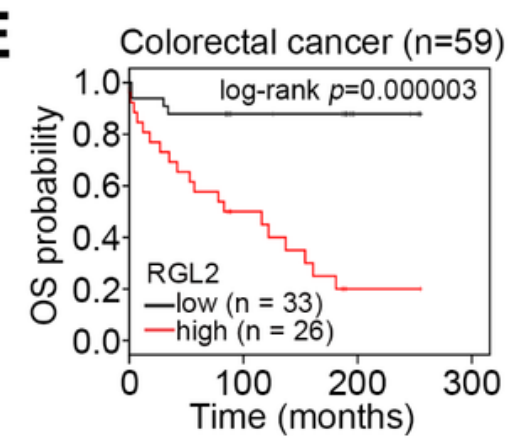

$F$

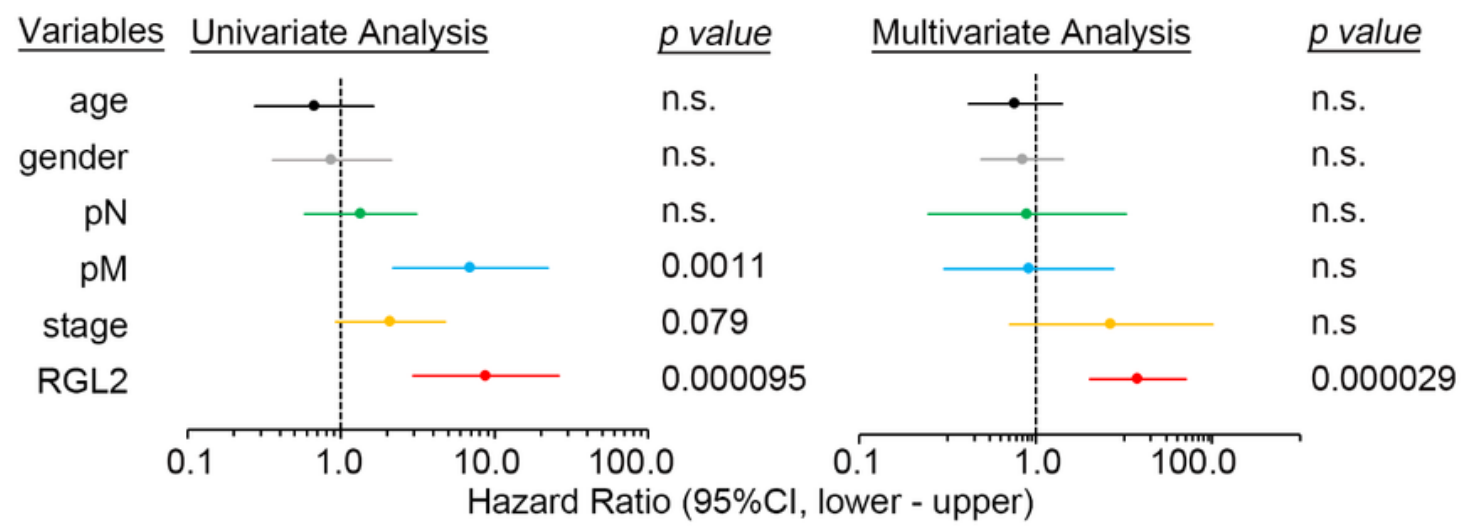

Figure 2

RGL2 upregulation refers to a poorer prognosis in colorectal cancer patients. (a) Forest plot for the hazard ratio (HR) of RGL2 (high expression v.s. low expression) in a meta-analysis using the PrognoScan database. Cox-p denotes the $p$ value obtained from the Cox repression test. (b and c) Kaplan-Meier 
analyses for RGL2 transcript using disease-free survival (DFS) and overall survival (OS) under a minimized $p$ value against CRC cohorts from the GSE17537/GSE17536 datasets (b) and the TCGA database (c). (d) Representative IHC scoring for the protein intensity of RGL2 (left) and the patient number in each IHC score (right). (e) Kaplan-Meier analysis for the low (scores 0 and 1 ) and high (scores 2 and 3 ) protein levels of RGL2 determined by IHC experiment against the enrolled CRC patients in the commercial tissue microarray. (f) Cox regression test using univariate and multivariate models for RGL2 protein levels (high vs. low) under OS conditions against the enrolled CRC patients in the commercial tissue microarray. 
A

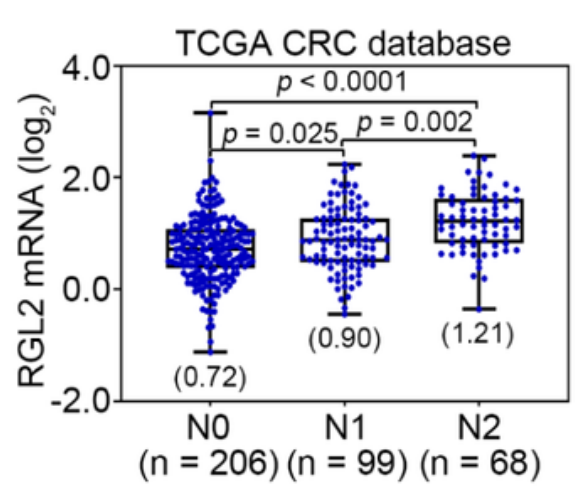

B

RT-PCR: DLD1 116 HT29 LoVo

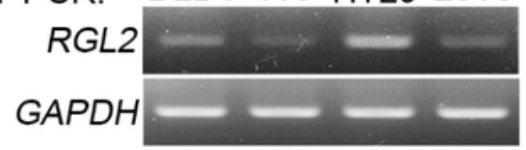

WB:

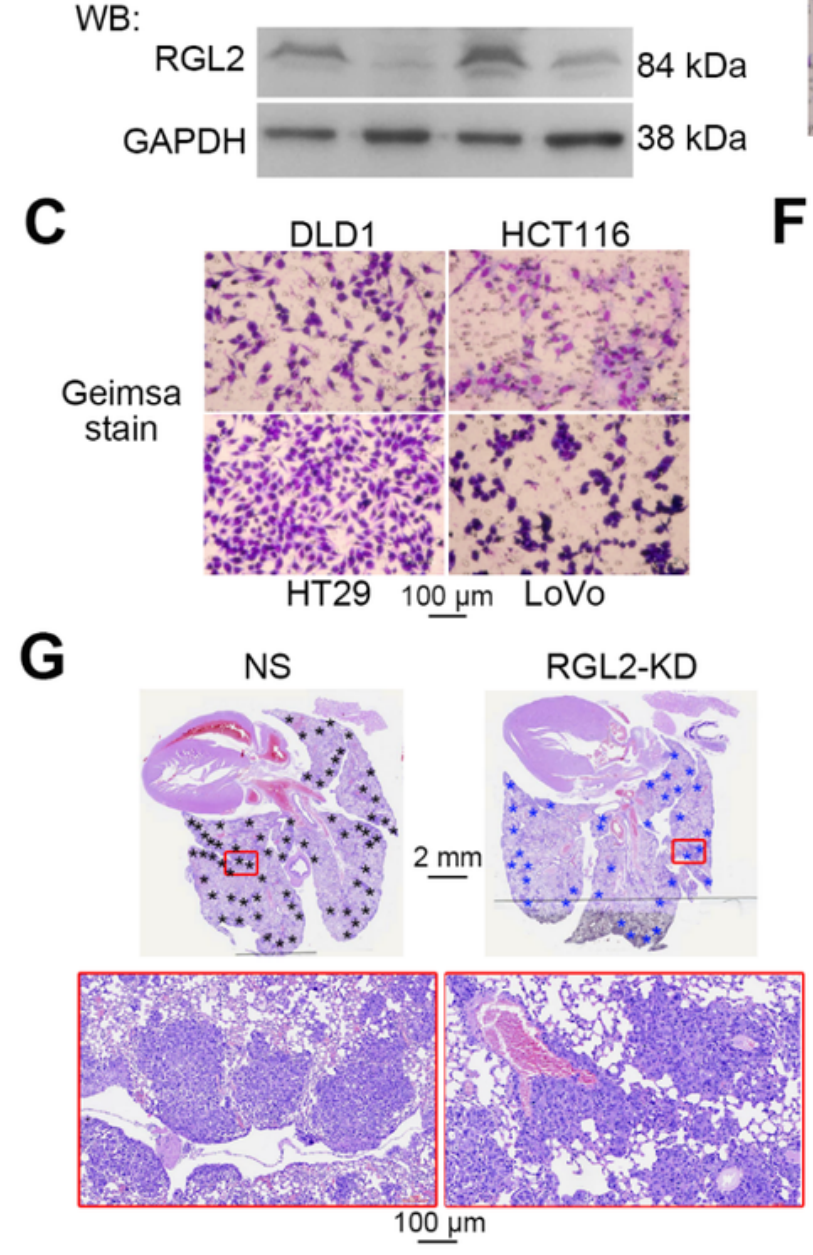

D

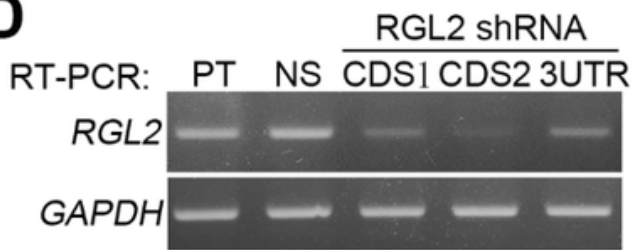

WB:

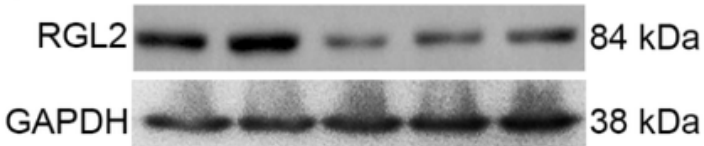

HT29

$\mathbf{E}$
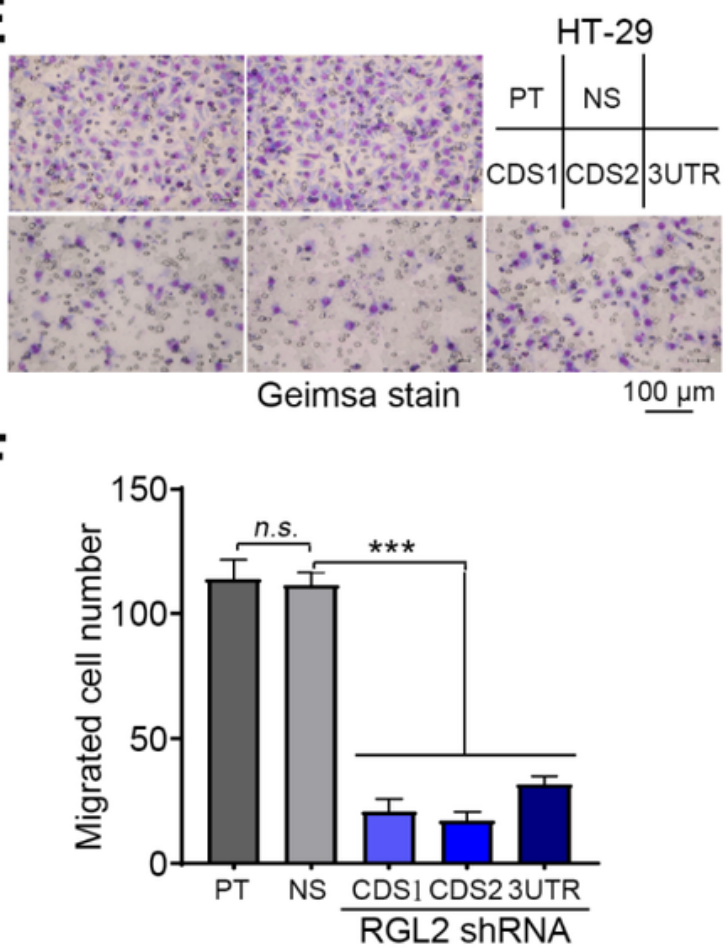

H

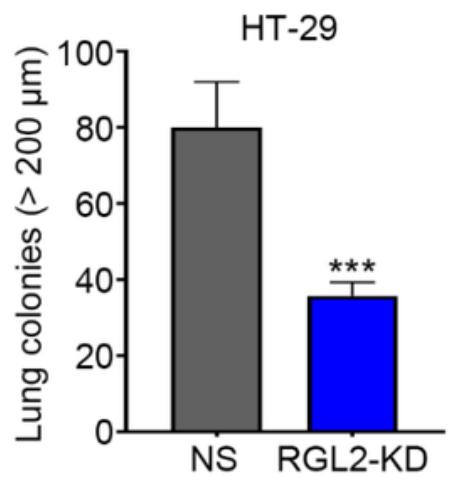

Figure 3

RGL2 expression is causally associated with the metastatic potential of CRC cells. (a) Boxplot for the transcriptional profiling of RLG in the TCGA CRC samples from patients with different pathologic $\mathrm{N}$ stages. (b) RT-PCR and Western blot analyses of the mRNA and protein levels, respectively, of RGL2 and GAPDH in the detected CRC cell lines. (c) Geimsa staining for the migrated cells in the Transwell cultivation of the detected CRC cell lines. (d) RT-PCR and Western blot analyses of the mRNA and protein 
levels, respectively, of RGL2 and GAPDH in the parental (PT), nonsilencing control (NS) and RGL2silenced HT-29 cell variants. In $b$ and d, GAPDH was used as the internal control for RT-PCR and Western blot experiments. (e and f) Geimsa staining (e) and histogram (f) for the migrated cells of the HT-29 cell variant shown in $\mathrm{d}$. ( $\mathrm{g}$ and $\mathrm{h}$ ) H\&E staining $(\mathrm{g})$ and histogram $(\mathrm{h})$ of the tumor colonies counted from the lungs of mice transplanted with NS and RGL2-knockdown (RGL2-KD) HT-29 cells.

A

B

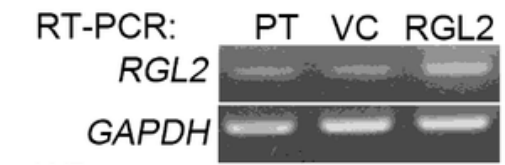

WB:

RGL2 $84 \mathrm{kDa}$ GAPDH

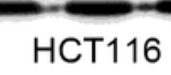

PT

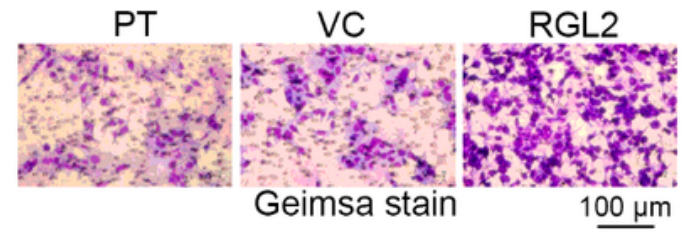

C

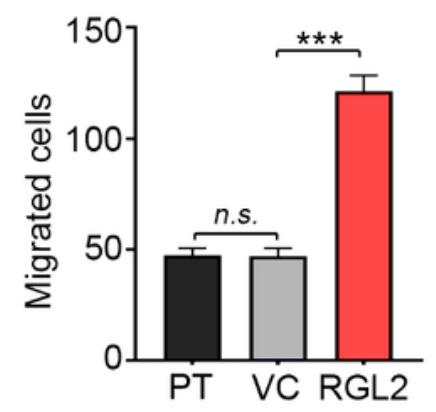

G
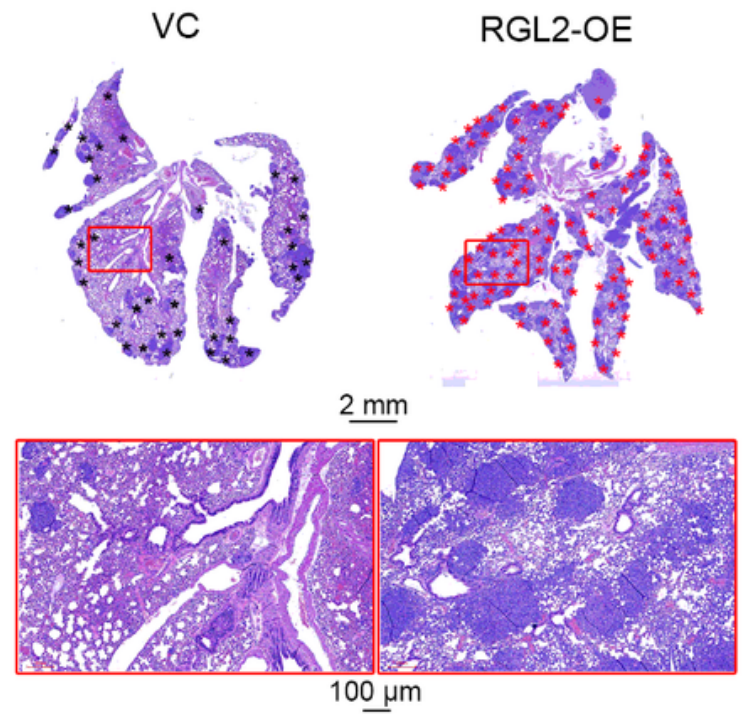

D

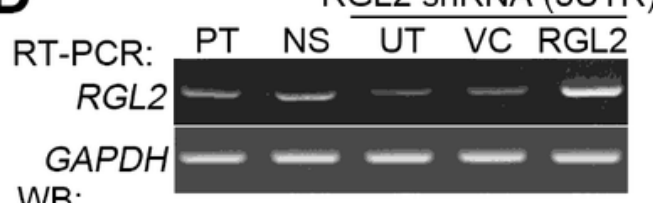

WB:

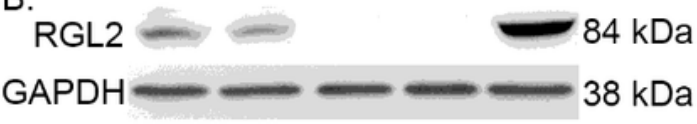

E

HT29
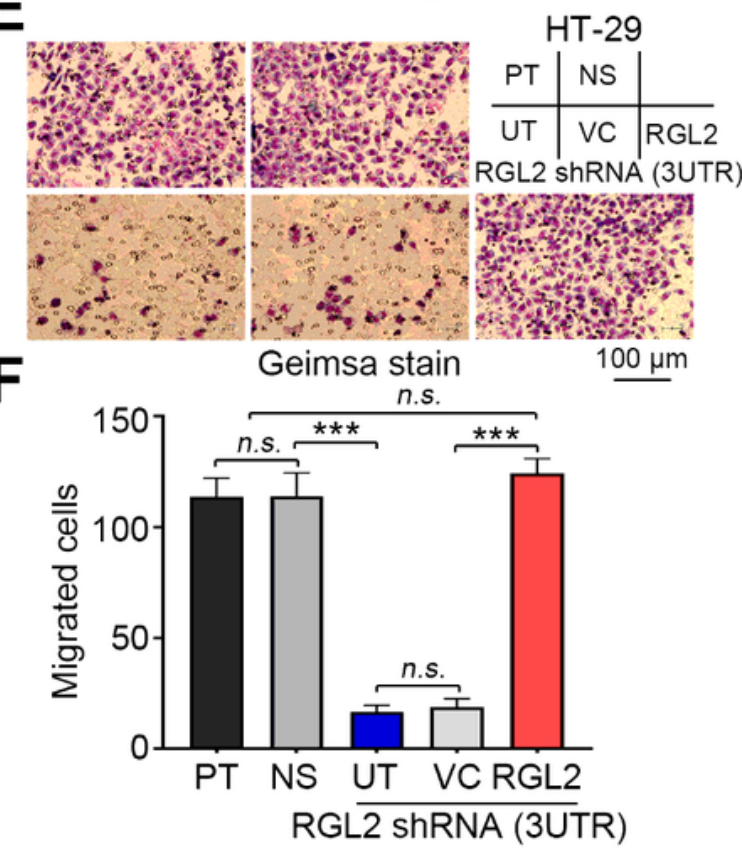

H

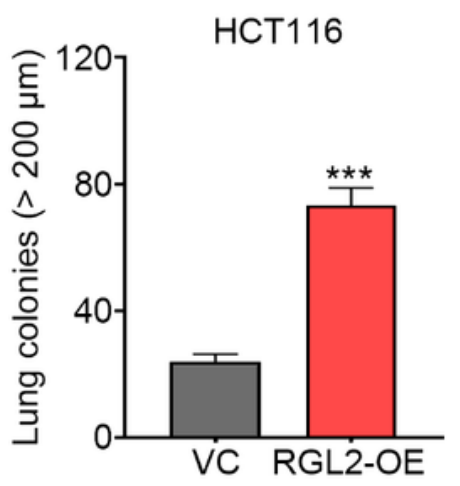

Figure 4 
RGL2 acts as a driver in promoting the metastatic progression of CRC cells. (a) RT-PCR and Western blot analyses of the mRNA and protein levels, respectively, of RGL2 and GAPDH in the parental (PT), vector control (VC) and RGL2-overexpressing HCT116 cell variants. (b and c) Geimsa staining (b) and histogram (c) for the migrated cells of the HCT116 cell variant shown in a. (d) RT-PCR and Western blot analyses of the mRNA and protein levels, respectively, of RGL2 and GAPDH in PT and NS control HT-29 cells and RGL2-silenced HT-29 cells without (untreated, UT) or with transient transfection of vector control or vector-containing RGL2 gene. In a and d, GAPDH was used as the internal control for RT-PCR and Western blot experiments. (e and f) Geimsa staining (e) and histogram ( $f$ ) for the migrated cells of the HT-29 cell variant shown in $d$. ( $g$ and $h$ ) H\&E staining $(g)$ and histogram (h) of the tumor colonies counted from the lungs of mice transplanted with VC and RGL2-overexpressing (RGL2-OE) HT-29 cells. 
$\mathbf{A}_{\text {TCGA_Colorectal cancer }} \mathbf{B}$

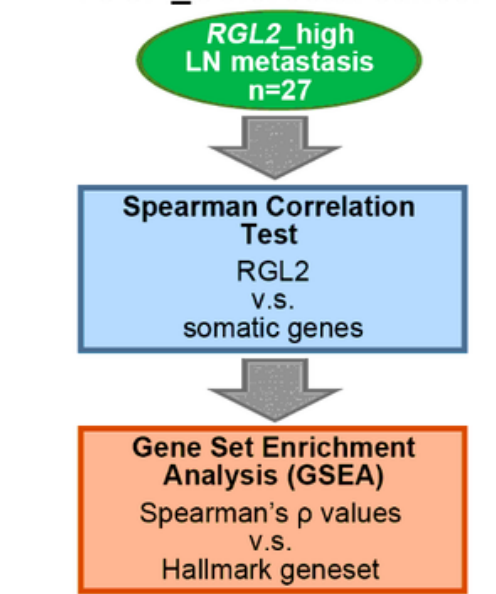

C

MSigDB_Hallmarks

WNT_Beta_Cätenin_Signaling Epithelial_Mesenchymal_Transition

UV_Response_DN

Apical_Junction

Myogenesis
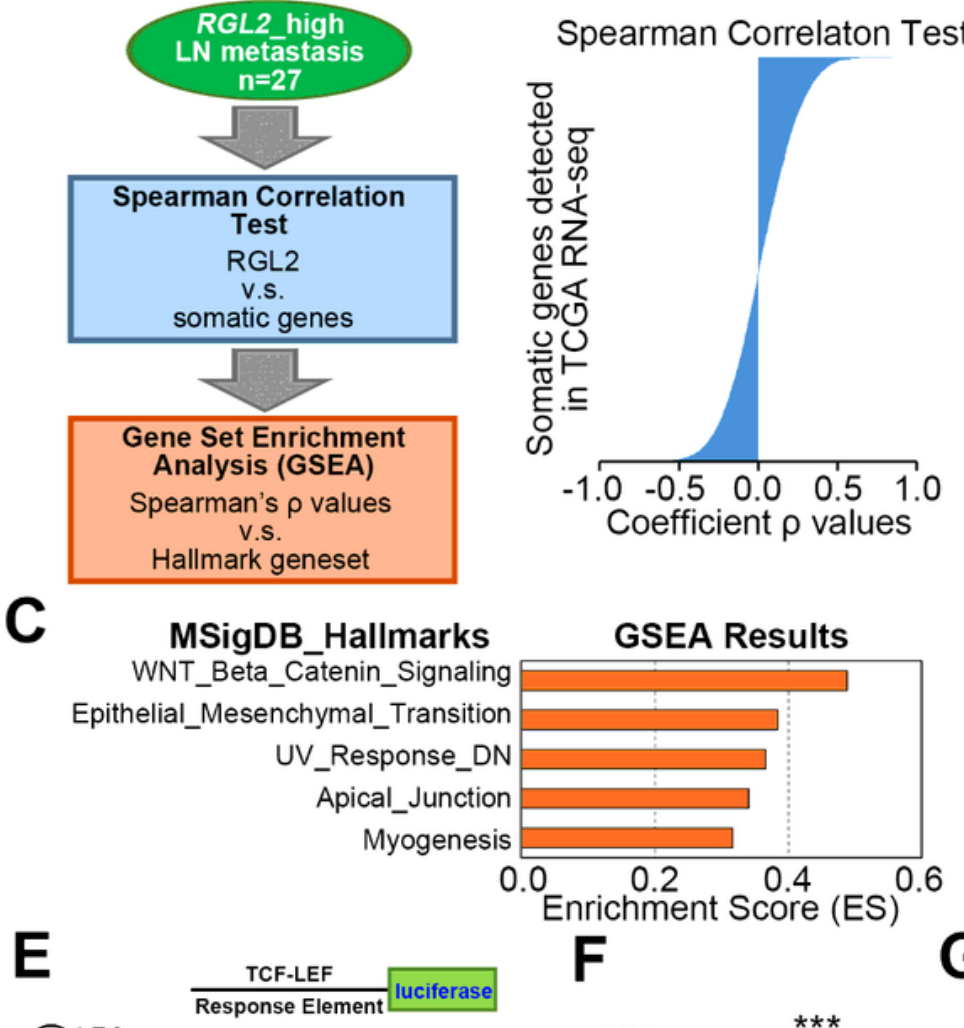

D

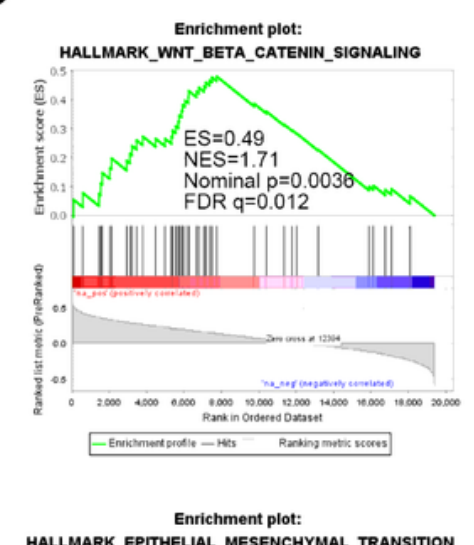

HALLMARK_EPITHELIAL_MESENCHYMAL_TRANSITION

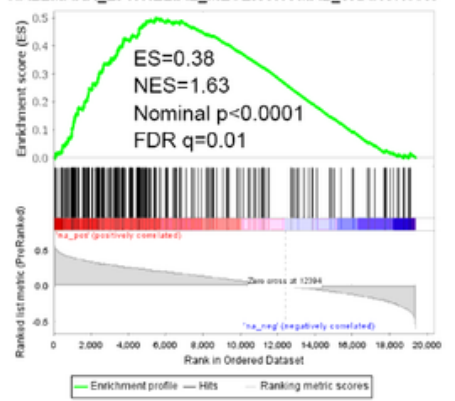

$\mathbf{F}$

\section{GSEA Results}

G

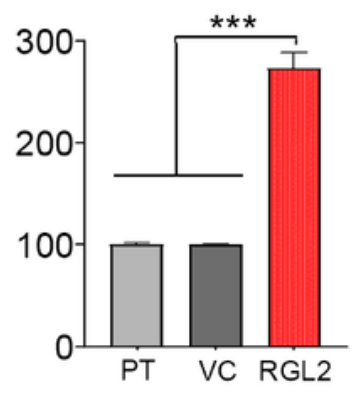

J

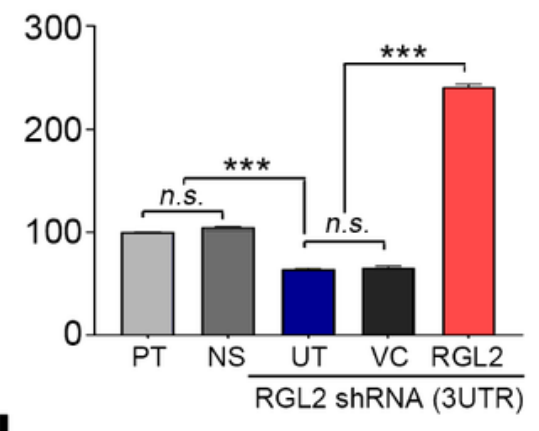

H
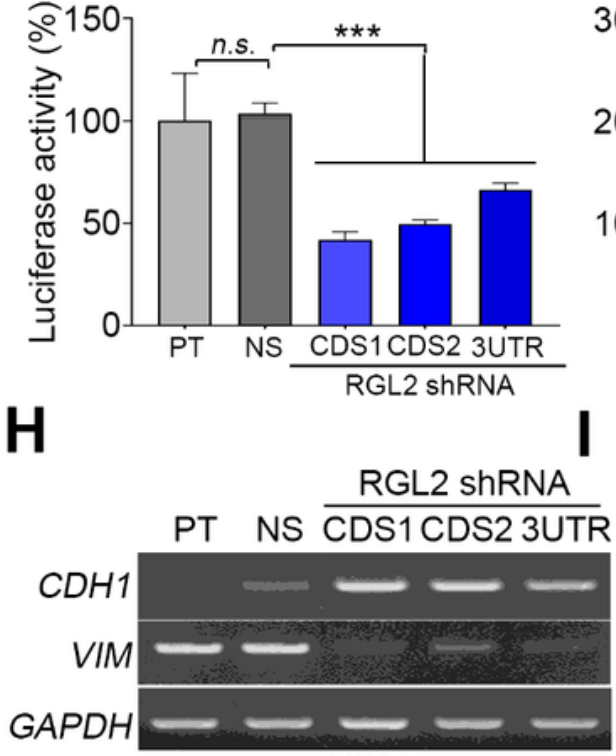

HT29

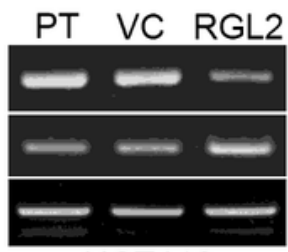

HCT116

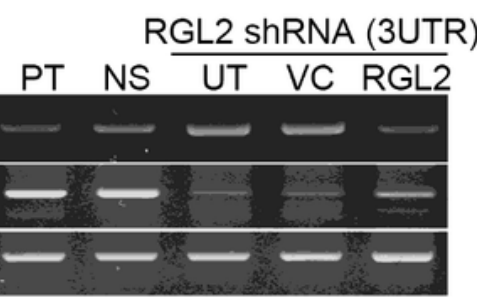

HT29

\section{Figure 5}

RGL2 upregulation correlates with the activation of the Wnt/ $\beta$-catenin signaling axis and EMT progression in CRC. (a) The flowchart of generating the RGL2 signature and performing GSEA simulation. (b) Spearman's coefficient $\rho$ values obtained from RGL2 coexpression with other somatic genes detected by RNA-sequencing experiments in the enrolled TCGA CRC samples. (c) Histogram for the enrichment scores of GSEA simulation against the RGL2 signature and hallmark gene sets. (d) The enrichment score 
(ES) derived from the correlation among the Wnt/ $\beta$-catenin and EMT gene sets and the queried Spearman's correlation coefficient $\rho$ is plotted (green curve). NES and FDR denote the normalized enrichment score and the false discovery rate, respectively. (e-g) Histogram for the luciferase activity, relative to parental cells, measured in the HT-29 (e and g) and HCT116 (f) cell variants transfected with luciferase reporter vector harboring a TCF-LEF response element for 24 hours. (h-j) RT-PCR experiments for the $\mathrm{CDH} 1$ and VIM mRNA levels in the indicated HT-29 ( $\mathrm{h}$ and j) and HCT116 (i) cell variants. GAPDH was used as an internal control of experiment.

A

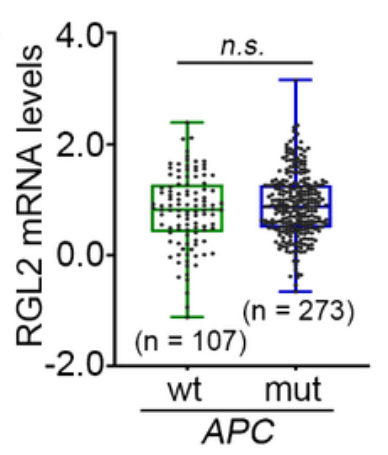

B

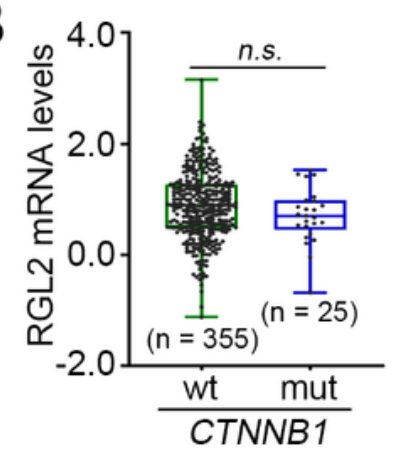

C

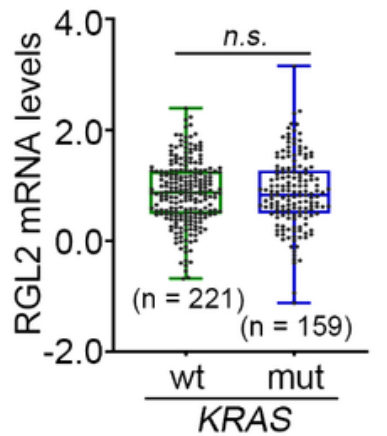

D

PT NS $\frac{\text { RGL2 ShRNA }}{\text { CDS1CDS2 3UTR }}$

k-Ras - - - - $-21 \mathrm{kDa}$

GAPDH

$38 \mathrm{kDa} \underset{(\text { Ser33/37/Thr41) }}{\mathrm{p}-\beta-\text { atenin }}$

VC:

H

$k-R a s$

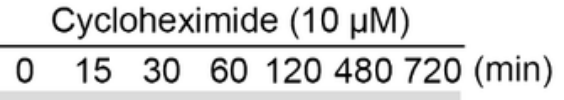

$0 \quad 1530 \quad 60120480720$ (min)

GAPDH $92 \mathrm{kDa}$

HT-29

RGL2-OE:

E

PT VC RGL2

k-Ras

$21 \mathrm{kDa}$

GAPDH

$38 \mathrm{kDa}$

k-Ras

p- $\beta$-catenin

(Ser33/37/Thr41)

GAPDH

HCT116

$\mathbf{F}$

RGL2 ShRNA (3UTR)

PT NS UT VC RGL2

k-Ras - - $-21 \mathrm{kDa}$

GAPDH $---\infty 38 \mathrm{kDa}$

HT-29

G

Cycloheximide $(10 \mu \mathrm{M})$

NS: \begin{tabular}{lllllll}
\hline 0 & 15 & 30 & 60 & 120 & 480720 & (min)
\end{tabular} k-Ras $21 \mathrm{kDa}$ p- $\beta$-catenin (Ser33/37/Thr41) $92 \mathrm{kDa}$ GAPDH $38 \mathrm{kDa}$

RGL2-KD:

k-Ras $--=-21 \mathrm{kDa}$ p- $\beta$-catenin (Ser33/37/Thr41) $92 \mathrm{kDa}$

GAPDH $38 \mathrm{kDa}$

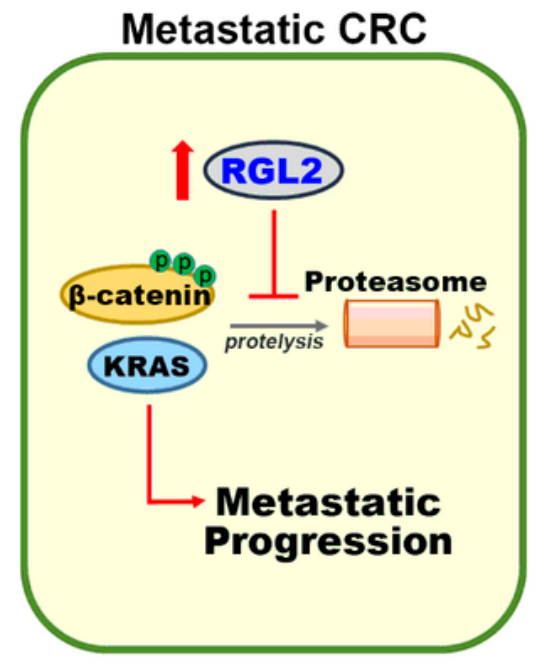

HT29 


\section{Figure 6}

RGL2 expression is irrelevant to KRAS mutation but regulates the protein levels of KRAS in CRC. (a-c) Boxplot for the transcriptional profiling of RGL2 in the TCGA samples without (wild-type, wt) or with (mutant, mut) APC (a), $\beta$-catenin (CTNNB1, b) and KRAS (c) mutations. (d-f) Western blot analyses for the protein levels of KRAS and GAPDH in the designated HT-29 ( $d$ and $f$ ) and HCT116 (e) cell variants. ( $g$ and h) Western blot analyses of the protein levels of KRAS, phosphorylated $\beta$-catenin at Ser33/37/Thr41 and GAPDH in cycloheximide (10 [M)-treated NS/RGL2-KD HT-29 (g) and VC/RGL2-OE HCT116 (h) cell variants at the indicated time intervals. In $\mathrm{d}, \mathrm{e}, \mathrm{f}, \mathrm{g}$ and $\mathrm{h}, \mathrm{GAPDH}$ was used as an internal control for protein loading. (i) An illustration of the proposed model for RGL2-promoted metastatic progression in CRC. 